\title{
Editorial
}

\section{Human Factors and Ergonomics in the Minerals Industry}

\author{
Robin Burgess-Limerick ${ }^{*} \S$
}

\section{Minerals Industry Safety and Health Centre, Sustainable Minerals Institute, The University of Queensland, Australia ${ }^{\S}$ Guest Editor}

It gives me great pleasure to present this collection of 6 papers for The Ergonomics Open Journal. The papers provide a glimpse of the range of Human Factors and Ergonomics issues which are currently relevant for the minerals industry. The paper authors are, in the main, from Australia, which is, arguably, leading the world in developing safe and productive technological solutions to operating in harsh mining environments.

The first paper provides an illustration of the way in which an analysis of injury narratives describing injuries associated with underground mining equipment can highlight opportunities for improving equipment design. In part, the paper provides an argument for the introduction of teleoperation and automation as an effective way of reducing risks of injury and fatality.

This theme is taken up by the second paper in the collection, in which Lynas and Horberry explore issues related to the automation of mining equipment, and draw on lessons learned in other industries. One of the key messages of this paper is that automation can lead to unanticipated consequences and that decisions about the optimal degree of automation require careful consideration.

In the third paper, James et al., similarly explore human factors issues associated with teleoperation and present empirical results from trials of the use of an immersive environment for teleoperation of a remote vehicle. In the

*Address correspondence to this author at the Minerals Industry Safety and Health Centre, Sustainable Minerals Institute, The University of Queensland, 4072 Australia; Tel +61 73366 4084;

E-mail: r.burgesslimerick@uq.edu.au fourth paper, Alem et al. address related issues aimed at understanding the human factors and technical requirements of providing a system to allow remote guidance of a local worker performing complex maintenance tasks such as may be required to support equipment located at remote mine sites.

The final two papers in the collection address different issues. Horberry et al. argue for the use of "Naturalistic Decision Making" approaches such as "Critical Decision Method" to understand the decisions made by operators in the minerals industry, and present two case studies to illustrate the utility of the approach in deriving information of great value in subsequent interface design activities.

Zupanc et al. examine a simulation of an existing mining vehicle interface which includes a known design flaw (alternating directional compatibility). Age related effects were observed in the ability to learn to operate the simulated equipment. The consequences of rectifying the design flaw was demonstrated to include a temporary increase in steering errors by participants who had practised with the original design, suggesting that altering equipment design requires care to avoid unanticipated consequences.

Collectively the papers provide an indication of the technological changes on the horizon for the minerals industry, and the important role which human factors and ergonomics researchers and practitioners have in ensuring that the technological advances integrate and leverage human abilities, preferences and limitations. 